\title{
Isolation and characterization of lectin from the Artist's Conk medicinal mushroom, Ganoderma applanatum (Agaricomycetes) and evaluation of its antiproliferative activity in HT-29 colon cancer cells
}

\begin{abstract}
The growth and lectin production of Ganoderma applanatum, a white rot fungus, was optimized in broth cultures. The fungus was found to have a higher growth rate and higher lectin activity when grown in a medium adjusted to $\mathrm{pH} 6.5$ at $26^{\circ} \mathrm{C}$ under stationary conditions. Expression of lectin activity started in 5-day-old mycelial culture; maximum activity was expressed after the 15th day of incubation. Among the various carbon and nitrogen sources tested, the carbon source sucrose and the nitrogen source yeast extract support maximum growth and lectin production. Lectin from G. applanatum was purified by ammonium sulfate precipitation and ion exchange chromatography. The purified fraction revealed a single band with a molecular weight of $35.0 \mathrm{kDa}$. Moreover, carbohydrates such as mannitol, glucose, sucrose, maltose, mannose, galactose, sorbose, and fructose were found to inhibit the hemagglutinating activity of the lectin. The purified lectins from $G$. applanatum contain cytotoxic and proapoptotic activities against HT-29 colon adenocarcinoma cells.
\end{abstract}

Keyword: Extracellular polysaccharides; Immunostimulation; Medicinal mushrooms; NK cell activity; Phagocytic assay; Splenocyte proliferation 